\title{
The Pivotal Role of Patient-centred Approaches in Successful Outcome of Evidence-based High Quality Cancer Care
}

\author{
Ahmed Nadeem Abbasi \\ Department of Oncology, The Aga Khan University Hospital, Karachi, Pakistan
}

Development of high quality evidence-based cancer service is a great challenge. Focusing the actual needs of cancer patients, which eventually translates into desirable patient outcomes, is only possible via a holistic approach adaptation during the planning phases of this service. The establishment of the cancer service is a teamwork. Treatment is only a component of the overall comprehensive cancer care and control plan.

Site-specific multidisciplinary team (MDT) tumor board is the foundation of cancer management. The multidisciplinary team works closely together, keeping patients' well-being in the centre in order to make a recommendation, which may not attract cent percent consensus among the experts, who are designated as the essential core site-specific specialists. In developing countries, this task is indeed an endeavour which requires voluntary commitment of all participating clinicians, who are conducting these site-specific MDT tumor boards, conducted on a non-mandatory basis. We shared our own experience of establishment of an independent non-institutional board, named as City Tumor Board, Karachi in a manuscript published in December 2013 in which audit findings were presented.1 In almost every single case discussed at the City Tumor Board, we end up discussing the solution of financial constraints, which majority of cancer patients living in low and middle income countries (LMIC) face right from day one of their treatment. We opt for evidence-based management option for our patients and wish to deliver it in a cost-effective and cost-efficient manner. Limited studies are being published in LMIC on the subject of patient-centred approaches in cancer management. Some inferences can be drawn from studies performed in the West, addressing the sections of population who resemble our social conditions; thus we can extract some meaningful relevant inferences from their original work. I would like to quote a study which was conducted in the Knowledge Institute of St Michael's Hospital, Toronto, Canada. Concept mapping was used as a tool

Correspondence to: Dr. Ahmed Nadeem Abbasi, Department of Oncology, The Aga Khan University Hospital, Stadium Road, Karachi, Pakistan

E-mail: nadeem135@gmail.com

Received: December 30, 2019; Revised: January 7, 2020; Accepted: January 7, 2020 in order to make a comparison of opinions made by stakeholders in the utilisation of screening in early detection of cancer in South Asian population. This is an example of learning from the scientific work done in the West on similar, if not the same, cohort of individuals who are our target population as well. South Asian population showed a different pattern of acceptance of these screening services. This inference needs to be studied further via more innovative studies. ${ }^{2}$

A comprehensive cancer care service revolves around a patient-centred approach. We follow international guidelines. These clinical practice guidelines, in the National Comprehensive Cancer Network (NCCN), is being revised and updated on a regular basis. In the 2019 NCCN guidelines, it is mentioned that it is being updated by 53 expert panels, each of which is being comprised of specialists who are involved in site-specific practice in 28 different NCCN member institutions. ${ }^{3}$

These clinical guidelines assume that a site-specific multidisciplinary expert team is in place which is deliberating and documenting recommendations for management plan of each and every case which is discussed in a mandatory tumor board. Once we establish a site-specific MDT tumor board, only then we can safely and confidently apply recommendations for treatment as per clinical practice guidelines. ${ }^{3}$

Concept mapping of a patient-centred approach can play a pivotal role in the strategic visioning of cancer service planning. ${ }^{2}$ In developing countries, the role and rationale of these site-specific MDT tumor boards get more imperative weightage. In one of our correspondence published in Journal of Clinical Oncology, we insisted on identifying these site-specific MDT meetings as lifeline of our cancer patients. 4

The more we emphasise on this patient-centred approach, the better for our quality of recommendations produced at the end of site-specific MDT tumor board deliberations. We can learn from any well-established healthcare system. As we cannot work in isolation, so we strongly recommend international exchange programmes, which are based on pre-defined learning objectives. In addition to clinical training, a leadership skill-development component is also required. International exchange programmes also play a facilitatory role in this regard. 5 
Further improvement in clinical outcome of cancer patients getting treatment in LMIC is heavily dependent on our commitments towards making a strategic teamplan for the cancer service development in a holistic manner, keeping in view the needs of our cancer patients. Conceptual modelling of patients' own experiences is also an innovative area which needs our attention for further learning and idea exploration. 6

Patient is the key beneficiary of any endeavour which is based on open and independent multidisciplinary approach. In our scenario, Tumor Board establishment is one of the best process-developments, which can be considered as a starting point of improvement in cancer care planning. This notion is more true in developing countries, which are still in the process of development of a national cancer plan. In developing countries like Pakistan and other LMIC, the implementation of most appropriate economic healthcare model will ensure financial sustainability of cancer care service. We do explore and consider cost-efficient ways in order to maximise our service scope and to offer treatment in a sustainable environment. The quest of achieving this goal of offering the best treatment to our cancer patients, as per international standards, is going on and will continue keeping in mind that this quest is a journey and not a destination. There are ways to assess the overall outcome of any such model at tertiary care service level. All components of cancer care service provision can be monitored and evaluated via clinical quality indicators. ${ }^{7}$ The guidelines published by the National Institute of Health and Care Excellence (NICE) of United Kingdom are also a source of knowledge, which can give directions to healthcare policy-makers. NICE guidelines cover directions pertaining to economic evaluation models as well. 8

In future, we would be witnessing an enhancement of our clinical acumen and understanding of better cancer care via development of a better patient-centered concept mapping based on a multidisciplinary team approach. The more we reflect on this approach, we can get the better outcome of treatment parameters. In fact, this improvement in outcome will definitely translate into a better professional accomplishment for the clinicians and the whole healthcare team involved in the comprehensive holistic care of cancer patients.

\section{REFERENCES}

1. Asghar AH, Abbasi AN, Jamal A, Haider G, Rizvi S. City tumor board Karachi: An innovative step in multidisciplinary consensus meeting and its two years audit. J Pak Med Assoc 2013; 63:1534-5.

2. Lobb $R$, Pinto $A D$, Lofters $A$. Using concept mapping in the knowledge-to-action process to compare stakeholder opinions on barriers to use of cancer screening among South Asians. Implement Sci 2013; 8:37.

3. National Comprehensive Cancer Network. 2019 clinical practice oncology guidelines. https://www.nccn.org/professionals/default. aspx [Accessed on 6 January 2020].

4. Abbasi AN, Karim MU, Ali N, Hafiz A, Qureshi BM. Multidisciplinary team tumor boards are a lifeline for our cancer patients in lower and middle income countries. Clin Oncol 2016; 28:799.

5. Karim MU, D Aviero A, Khan AMH, Abbasi AN. Importance of international exchange program in postgraduate training. J Coll Physicians Surg Pak 2018; 28:981-2.

6. Herman JM, Kitchen H, Degboe A, Aldhouse NVJ, Trigg A, Hodgin $\mathrm{M}$, et al. Exploring the patient experience of locally advanced or metastatic pancreatic cancer to inform patientreported outcomes assessment. Qual Life Res 2019; 28: 2929-39.

7. Clauser SB, Johnson MR, O'Brien DM, Beveridge JM, Fennell ML, Kaluzny AD. Improving clinical research and cancer care delivery in community settings: Evaluating the $\mathrm{NCl}$ community cancer centers program. Implement Sci 2009; 4:63.

8. National Institute for Health and Care Excellence, United Kingdom. https://www.nice.org.uk. [Accessed on 6 January 2020]. 